\title{
Tactile Rendering of 3D Features on Touch Surfaces
}

\author{
Seung-Chan Kim ${ }^{1,2}$, Ali Israr ${ }^{1}$, Ivan Poupyrev ${ }^{1}$ \\ ${ }^{1}$ Disney Research Pittsburgh, \\ 4720 Forbes Avenue, \\ Pittsburgh, PA, 15213 USA \\ \{kimsc, israr, ivan.poupyrev\} \\ (a)disneyresearch.com \\ ${ }^{2}$ Department of Mechanical Engineering, \\ KAIST \\ 291 Daehak-ro, Yuseong-gu, Daejeon \\ 305-701, Korea
}

\begin{abstract}
We present a tactile-rendering algorithm for simulating $3 \mathrm{D}$ geometric features, such as bumps, on touch screen surfaces. This is achieved by modulating friction forces between the user's finger and the touch screen, instead of physically moving the touch surface. We proposed that the percept of a 3D bump is created when local gradients of the rendered virtual surface are mapped to lateral friction forces. To validate this approach, we first establish a psychophysical model that relates the perceived friction force to the controlled voltage applied to the tactile feedback device. We then use this model to demonstrate that participants are three times more likely to prefer gradient force profiles than other commonly used rendering profiles. Finally, we present a generalized algorithm and conclude the paper with a set of applications using our tactile rendering technology.
\end{abstract}

ACM Classification: H5.2 [Information interfaces and presentation]: User Interfaces. - Graphical user interfaces, Input devices and strategies, Haptic I/O.

Keywords: Electrovibration, haptic rendering, friction

\section{INTRODUCTION}

Touch and gesture interactions have rapidly proliferated in recent years and have become a de-facto standard in mobile phones, tablet and desktop computers. Consequently, the efforts to develop effective, feasible and inexpensive tactile feedback for touch screen interaction have also increased $[15,19]$. The goal of this work is to develop tactile rendering techniques that provide rich, immediate and dynamic tactile feedback to users interacting with touch screens. Such tactile feedback not only increases the effectiveness of touch interactions, but also leads to more realistic and satisfying experience for millions of users.

Early pioneering work on touch screen tactile feedback focused on using mechanical vibrations, where the screen or the entire device would move rapidly to stimulate a user's

Permission to make digital or hard copies of all or part of this work for personal or classroom use is granted without fee provided that copies are not made or distributed for profit or commercial advantage and that copies bear this notice and the full citation on the first page. Copyrights for components of this work owned by others than the author(s) must be honored. Abstracting with credit is permitted. To copy otherwise, or republish, to post on servers or to redistribute to lists, requires prior specific permission and/or a fee. Request permissions from Permissions@acm.org. UIST'13, October 8-11, 2013, St. Andrews, United Kingdom.

Copyright is held by the owner/author(s). Publication rights licensed to ACM.

ACM 978-1-4503-2268-3/13/10...\$15.00.

http://dx.doi.org/10.1145/2501988.2502020 fingertip $[8,20]$. Challenges in designing feasible vibrotactile touch interfaces included cost, reliability, actuator size, power requirements, as well as difficulty in the effective control of the device's mechanical vibrations $[2,16]$.

Recently, a promising new direction in touch screen haptics has emerged. Instead of creating vibrotactile stimulation on a user's finger, it suggests modifying friction between a sliding finger and the touch screen surface $[2,11,29]$. It has been demonstrated that such devices allow for the creation of not only a feasible and lightweight tactile feedback apparatus suitable for touch screens, but also provide rich, dynamic and highly satisfying user experiences [2]. These friction displays are particularly suitable to modern touch and gesture interfaces that are based on sliding and flicking fingers on screen.

Despite the rich interaction opportunities that these displays provide, there has been little work done on designing and evaluating fundamental techniques and algorithms for generating rich tactile sensations on friction-based tactile displays. Much of the previous work has focused on the hardware and materials required to build such tactile devices [1, $2,29]$. Levesque et al. $[10,11]$ used a squeeze-film effect to modulate the friction forces between the user's finger and a thin glass plate, where the level of force feedback was proportional to general mathematical functions, such as sinusoid profiles, and their spatial densities. It showed effective-

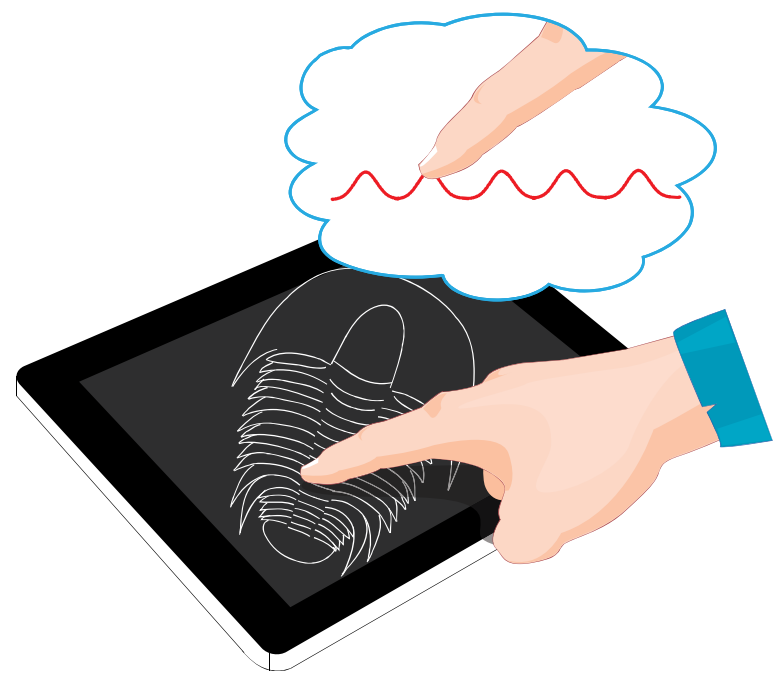

Figure 1: Tactile bump-rendering algorithm would allow the user to experience rich tactile textures on flat touch screens. 
ness of friction-based feedback displays in designing user interfaces; however, it did not attempt to convey fine 3D geometric details of the content presented to the user.

In this paper we propose, develop and evaluate an algorithm to render 3D geometrical features on friction-based tactile displays. The algorithm is based on an observation that the haptic perception of 3D features on flat surfaces depends predominantly on lateral forces applied on a finger $[17,21]$. We formulate a perceptual model that relates the perceived strength of friction as a function of the voltage applied to a friction display. We then utilize this model to modulate the perceived friction levels and render differentiating tactile feedback for complex 3D objects; such as textures, facial features, 3D models, surface forms and topographies, etc. Although the proposed algorithm is applicable to all lateral force feedback devices, our development is based on electrovibration-based tactile feedback.

We believe that the development of our tactile rendering algorithms expands the vocabulary of possible tactile representations and allows to render rich tactile information overlaid on visual content. This will lead to new exciting uses of tactile displays technology for touch screen interaction.

\section{BACKGROUND AND RELATED WORK}

Given a haptic feedback apparatus, creating a realistic and compelling tactile representation of objects and interactions is an important challenge in haptic research that is not well understood (see e.g., [16]).

One fundamental direction in creating such "tactile content" includes the formation of a set of abstract "tactile primitives" that are perceptually differentiable and can be easily learned and recognized. For example, in 1957 Frank Geldard developed "Vibratese language" that consisted of five vibrators placed at five locations on the back [5]. Variations in location, intensity and timing of the vibrations were coded to represent alphanumerical characters. In more recent work, tactile primitives have been assigned to provide feedback for various interaction events $[3,8]$. These tactile primitives were mainly defined for the vibrotactile stimulation and are not scalable to friction-based displays.

Another direction in haptic content generation is the simulation of the dynamic physical properties of objects and environments. Typically, force-feedback devices are used to render inertial, stiffness and viscous elements of simulated objects and environments [17, 23]. "Record and Play" strategies have also been used, where physical features of the object were first measured and then re-played at a later time when the user interacted with virtual objects [22]. These techniques are applicable when the physical deformation models of objects are either available or can be measured. However, in cases where a finger slides over an arbitrary texture, the contact conditions cannot be empirically determined.

Finally, an emerging direction in haptic content generation techniques focuses on affective haptic events that neither simulate nor provide information per se, but attempt to communicate a feeling, a mood or a special effect. For ex- ample, Yohanan and MacLean [30] used "affective" models to modulate user emotions while users interact with a haptic apparatus. Israr and Poupyrev [9] developed psychophysical models to create, control and modulate a feeling of motion on human skin, resulting in novel feelings and effects that could not be experienced otherwise.

It is important to emphasize that all of these techniques are hardware specific and do not usually scale well either to other modalities of touch perception or to other haptic apparatus. The design of tactile feedback content is invariable tied to the character of haptic actuation. For example, the perceptual characteristics of vibrations cannot be applied to force-feedback applications. Similarly, techniques defined for force-feedback devices cannot be applied to frictionbased interactions where the user's finger is actively sliding and exploring the surface of a touch screen.

Most modern friction haptic displays are based on two basic technologies. In tactile displays based on a squeezefilm effect, a thin sheet of glass placed on top of the screen is laterally vibrated at an ultrasonic frequency, creating a thin cushion of air under the touching finger $[4,29]$. By modulating the frequency and intensity of vibrations, this technology allows for a decrease in physical friction between the glass and sliding finger, making the screen "smoother" on demand.

In tactile displays based on an electrovibration effect the friction between the sliding finger and the touch screen is produced by injecting a periodic electrical signal into a conductive electrode coated with a thin dielectric layer [2]. The periodic signal creates an alternating electrostatic force that periodically attracts and releases the finger from the touch surface, producing friction-like rubbery sensations. Varying the frequency and amplitude of the periodic signal varies the quality of sensations along the stick-slip and smooth-rough continuums [2].

The advantage of using electrovibration-based lateral haptics in touch screen interaction is that it can be easily integrated into popular capacitive or optical touch screens and is inexpensive and lightweight. The nature of touch itself is also different - unlike in traditional haptic devices where the stimulus is presented to passive skin, the interactions on the friction tactile displays are "active", where the human hand freely moves on the screen, similar to our natural way of exploring surfaces and textures on real world objects [13]. Furthermore, friction-based displays are expressive and allow for the production of a rich palette of tactile sensations. This is because the frequency bandwidth of these devices is significantly broader than that of traditional electro-mechanical actuators [2].

This paper explores the design of tactile rendering algorithms that allow us to systematically generate rich and effective tactile sensations for lateral friction displays. We believe that the formulation, development and evaluation of such a tactile rendering algorithm are important contributions in the further development of practical and useful tactile displays for touch screen interactions. 


\section{TACTILE RENDERING ON A FRICTION DISPLAY}

The fundamental observation that is used in designing the tactile rendering algorithm is that a perception of a physical bump by a finger sliding on a physical surface is defined predominantly by lateral forces applied to the finger. This hypothesis was initially explored as early as in 1990 [17] and it was experimentally validated in perceptual studies conducted using active forces by Robles-De-La-Torre and Hayward in 2001 [21]. This is both a highly significant fact and an exciting discovery because it means we can create a perception of $3 D$ tactile features on a flat touch surface by manipulating only lateral forces, such as friction. In other words, friction-based displays can create tactile percepts of 3D bumps on touch screens.

The algorithmic tactile rendering of a 3D bump on a touch screen opens up a broad range of exciting applications for the tactile display of images, videos and interactive computer graphics on touch screens. This includes, but is not limited to geometric features such as ridges, edges, protrusions, textures, etc.

The rest of the paper is organized as follows. In the next section we discuss what a tactile bump is and how users perceive it. We discuss experimental studies of bump perception in detail, in particular, the effect of lateral forces. We then continue the discussion of techniques for rendering 3D bumps using electrovibration-based friction-based tactile displays. The design of the algorithm consists of two parts. First, we design and investigate a perceptual model that links voltages that are used to control friction to the subjective friction strength. By using this model we can control the perceived strength of friction, which can be useful broadly beyond the scope of this paper. The development and evaluation of this model is one contribution of this paper.

Second, we propose and evaluate a lateral force profile that creates a perception of the $3 D$ tactile bump. The proposed force profiles extend the previous work $[17,21]$ that we have discussed above. We conduct an experiment that demonstrated the effectiveness of lateral friction profiles compared to two other general control schemes. The design and evaluation of control schemes for rendering $3 \mathrm{D}$ tactile bumps forms the second contribution of the paper.

Finally, we illustrate the use of developed techniques to algorithmically generate tactile profiles for a variety of visual content. Specifically, we discuss a one-dimensional case to generate real world patterns, such as corduroy; the repeating objects, such as book stacks and others. We then generalize our algorithm to a two-dimensional case and demonstrate how it can be used to provide tactile feedback for any depth-based imagery, such as 3D computer graphics renderings or real-time images from a depth camera, such as Kinect. Designing such algorithms is the third contribution of this paper. An overview of potential applications concludes the paper.

\section{PERCEPTION OF BUMPS}

Before designing algorithms it is important to discuss what haptic bumps are and the mechanisms of their perception.
The human perception of small geometric features such as a bump is often categorized according to object scale, i.e., as features that fit under the fingertip or features with contours that extend beyond the fingertip.

The features that fit under fingertips are often referred to as tactile textures. They are perceived through static skin identation and require little finger motion [12]. When the feature has relatively large contours it cannot be perceived via a static finger and requires active haptic exploration [6] where forces perceived through touch and kinesthetic feedback from the moving finger are temporarily integrated. As the scale of the features increases larger motions are required and eventually also involve wrist and arm movements. In this paper, we are focusing on a medium-scaled geometric feature that can be perceived with only the motion of the wrist. We define such surface features as "bumps".

\section{Lateral forces for rendering geometric features}

The easiest approach to create the perception of bumps is to simply push or pull the user's finger by means of physical actuation using a vibrator [20], force feedback device [25], or electromagnetic device [27]. This approach, however, is often impractical when it comes to touch screens, as additional movement of the screen and force feedback devices is required.

A number of previous studies have demonstrated that variations in a lateral, horizontal force component experienced while sliding the finger over the surface can provide sufficient information regarding the surface geometry [17, 21]. Moreover, Gordon and Morison showed that the gradient, defined by dividing the base-to-peak height by half the surface length, is an effective stimulus for curvature perception, and humans rely on local curvature when perceiving surface $[7,14]$.

We can conclude that the surface slope or gradient at the touch point is the most important feature in perceiving curved objects [7]. This has been used early for simulating local geometric features such as bumps with haptic devices. For example, Minsky et al. [17] created an illusion of bumps by calculating the local spring force based on the local gradients of the virtual surface experienced by the user equipped with force-feedback joystick. Based on the observation that the steeper slope of bumps produces stronger resistance, previous researchers utilized slope information when creating a force field for simulating bumps on the surface [21]. A similar approach was also employed by a recent study that calculated the lateral force field based on the gradient of the image intensity function [24]. It is important to note that the earlier research discussed above based on using active force feedback devices and usually does not scale to other tactile feedback technologies, such as electrovibration-based tactile displays used in this paper.

\section{Rendering tactile bumps on touch screens}

Creating geometric features, such as bumps, on a flat touch screens without physically moving the touch panel under the user's fingertip has not been explored before. Here, we create a percept of tactile bumps by varying the frictional forces 


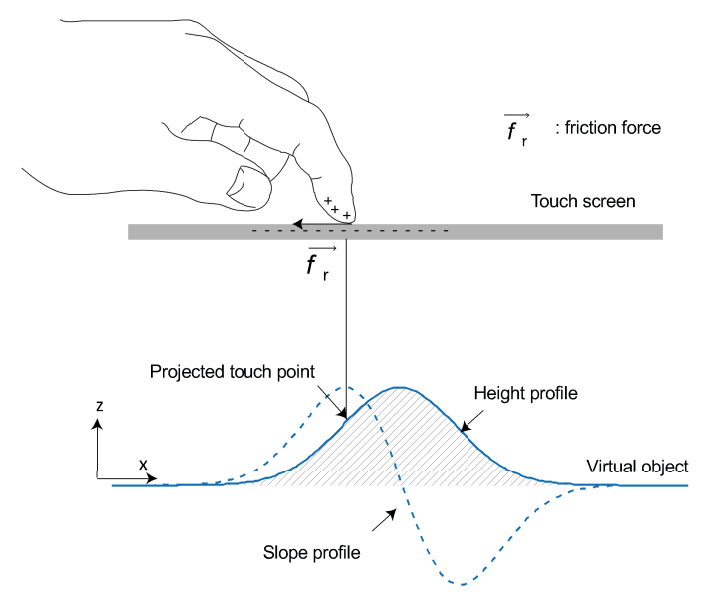

Figure 2: The use of electrovibration to create friction between the finger and the surface.

felt on the surface of the panel by the user's fingertip. More specifically, we propose that resistive forces on the fingertip are proportional to the local gradient of the geometric shape displayed on a touch screen, i.e., the steeper the bump - the higher the resistance forces felt on the finger. We create the perception of $3 \mathrm{D}$ surface bumps by modulating the resistance forces as a function of the virtual slope that the user interacts with (Figure 2).

We utilize an electrovibration tactile feedback devices, where the resistive forces felt by a user are a function of the voltage applied to the display [2]. Therefore, the voltage is the main control variable.

\section{Controlling perceived friction forces by electrovibration} In this section, we determine a psychophysical model describing relationship between perceived friction felt by the user and applied voltage.

Apparatus. An electrovibration display similar to the one in Bau et al. [2], was used in this study. The display consisted of a 3M Microtouch surface-capacitive panel used both to track the location of the sliding finger as well as to render haptic feedback on the panel. A simple transistor-based circuit, interfaced via a serial port, was used to control the frequency and amplitude of the voltage applied to the panel. The panel was placed in a wooden frame and connected to a laptop (Figure 3).

Methods. Eleven participants (six males, average age: 23.9 years) took part in the experiment. Experimental procedures for determining subjective force magnitude scales were similar to those presented in $[28,31]$. In each trial, participants scanned the touch panel with the index finger and felt the resistance corresponding to the test stimulus applied to the panel electrode. In order to obtain subjective scales for most common interactions on touch-screens, they were instructed to move their index finger freely in approximate linear strokes, thus not constraining their gestures by contact force, direction, velocity and length of stroke. Participants were asked to assign a number between 0 and 100 to describe the subjective friction intensity. Smaller numbers correspond to lower perceived friction.

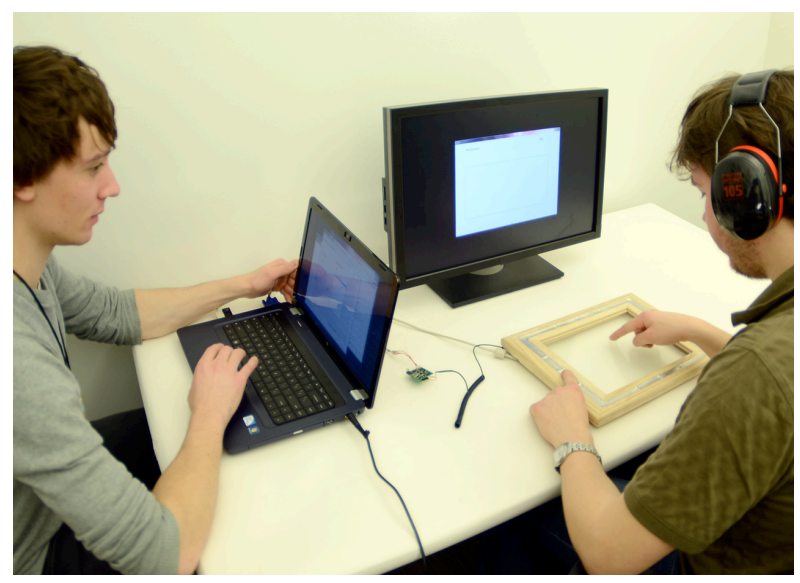

Figure 3: Experimental apparatus and setup.

Subjective friction scales for pure sinusoidal input voltage were determined at three test frequencies, namely 60,110 and $200 \mathrm{~Hz}$. The stimulus set consisted of ten equally spaced amplitude levels (in the logarithmic scale) at each test frequency. The ratio of two consecutive amplitude levels was set at 1.15 , that is a 15 percent increment from the lower level corresponding to $1 \mathrm{JND}$ apart between the two levels [2]. The lowest amplitude was 15 percent above the rough estimation of the absolute detection threshold at each test frequency. The entire stimulus set consisted of 30 test stimuli randomly presented three times in a test session. Each participant completed three test sessions of 90 trials, each with 5-10 minute intersession breaks. The total number of trials in the experiment was 2,970. Before the main experiment, participants familiarized themselves with the device, procedures, and rating scales. They wore earmuffs to block any environmental and/or device noise.

Results and Discussion. In order to reduce inter-participant variability, the raw subjective ratings were normalized by dividing them by the geometric mean of the ratings in the corresponding session and then multiplying the result by the overall geometric mean of the ratings obtained in the entire experiment.

Figure 4 shows normalized force ratings plotted against the voltage applied to the electrovibration display in the log-log scale, a standard way of plotting human subjective scales [28]. Each data point represents the average friction rating assigned to the voltage level by eleven participants, and the error bar represents the standard error of the mean. The entire data is regressed against a straight-line function, represented as a thick dashed line, to determine the best-fit line function between subjective ratings and applied voltage. The straight-line functions yielded high correlation coefficients ( $R$, degree of freedom, $d f=2968)$ of 0.69 .

The straight-line fit is the first order psychophysical model to relate the applied voltage amplitude and the perceived friction forces, and is estimated in the log-log scale as:

$$
Y=1.24 \cdot X-1.05
$$

The estimated model of perceived friction, i.e. the dashed line in Figure 4, is essential in the algorithm to render 3D 


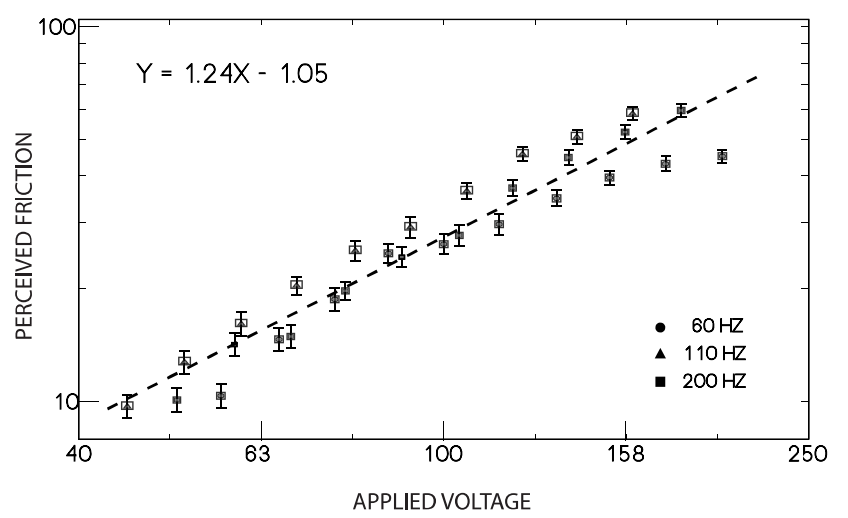

Figure 4: Subjective friction magnitude scales as a function of the voltage applied to the electrovibration-based lateral tactile feedback device.

surface features, such as bumps, holes, ridges and edges. Using the relationship, we can design and implement arbitrary friction force profiles on flat touch screen surfaces. Importantly, this relationship can be scaled to other configurations of electrovibration devices by adjusting the coefficients of Equation 1 according to the hardware specifications, such as thickness of dielectric layer and operating frequency.

\section{Pairwise comparison of lateral force profiles}

The main element in our tactile rendering procedure is the choice of a force profile that maps local geometrical properties of the virtual surface to friction forces. We conducted a pairwise comparison study to establish subjective preference of force profiles for rendering 3D tactile bumps on a touch screen. Three rendering profiles (S1, S2, S3) were computed for the Gaussian bump and compared (Table 1).

Height profile (S1) maps the depth of the surface geometry from a reference plane, which is usually a touch screen, to friction force. This method is often called displacement mapping and is used in force [25, 26] and tactile [29] rendering.

Slope profile (S2) maps the slope of the height profile to friction force adjusted for the direction of finger motion [21].

Rectangular profile (S3) is the most traditional binary on/off profile that has often been used in touch screen tactile feedback interfaces $[2,10]$.

A standard Gaussian-shaped virtual bump was used in this study, and the goal was to create its tactile representation that would feel like a real, physical bump. The friction force profiles were calculated as a function of displacement along the bump $x$ dimension (Table 1), here $A$ is the height, $m$ is the center, and $\sigma$ is the width of the virtual bump. The computed friction forces ranged from 1 and 100 in order to match the dynamic range of the electrovibration tactile device. They were then mapped to control voltages using the straight-line perceptual model defined in Figure 4.

Procedures. The same experimental setup was used as in the previous experiment, except that the tactile touch panel was secured directly on top of the monitor screen and placed horizontally on the table to synchronize haptic feedback with visual contents (Figure 5). Ten participants (6

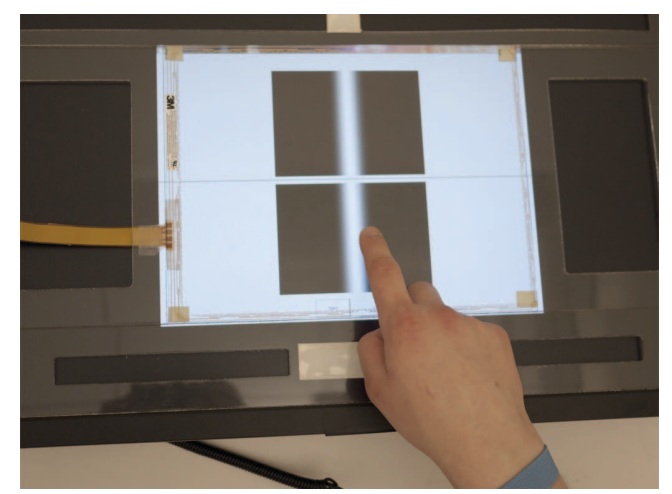

Figure 5. System configuration for the experiment.

males, average age: 26 years) took part in the study. A classic two-interval forced-choice procedure was used to obtain subjective preference. In each trial, participants were presented with two visual representations of bumps. Each bump was overlaid with one of the tactile-rendering profiles S1, S2 or S3. Participants were asked to choose the force profile that most resembled the real physical bump. Two widths ( $\sigma=20$ pixel and $\sigma=30$ pixel) and two heights ( $A=1$ and $A=0.8$ ) were randomly chosen for each profile pair to increase the variety of profiles in comparison trials.

The experimental session was divided into two blocks of 60 trials, separated by a 5-minute break. In each block, 12 comparison pairs ( 3 profile pairs $\times 2$ widths $\times 2$ heights) were presented 5 times in a random order, thus resulting in a total of 120 trials per participant. The slope profile $(S 2)$ was compensated to account for the direction of the finger's motion on the bump. The operating frequency for all profiles was set to $60 \mathrm{~Hz}$.

Results and Discussion. The proportion of preferred tactilerendering profiles is shown in Figure 6. Three pairs of bars represent the three possible pairwise combinations of profiles. Chi-square tests showed that participants' preferences in all comparisons were statistically significant $(p<0.01)$ regardless of the width and height variance $(p>0.05)$.

Results of the comparison experiments show that 1) participants preferred both the slope (85\%) and the height $(81 \%)$ profiles over commonly utilized rectangular profiles, and 2)

\section{Geometry to friction force mappings}

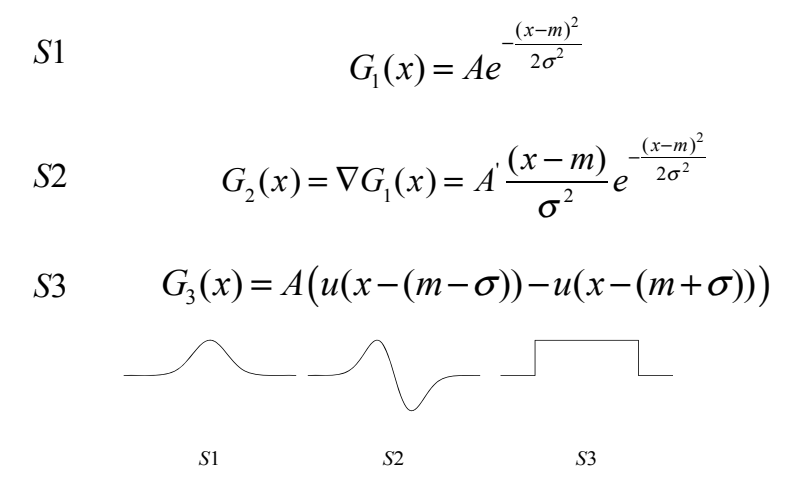

Table 1: Force rendering profiles 
participants preferred slope profiles $(73 \%)$ to the height rendering profile when compared in same trials.

\section{TACTILE RENDERING ALGORITHM}

In this section, we present an algorithm that generates tactile rendering of visual contents on friction-based lateral tactile displays. We first explain the algorithm in a one-dimensional case and then generalized it to a two-dimensional case.

Figure 7 summarizes the rendering algorithm. The input to the algorithm is a geometric representation of a surface to be rendered and the position and direction of motion of the finger sliding on the touch panel. The algorithm first computes the gradient of the surface based on the direction of the user's finger motion, it then determines the amplitude of the voltage that has to be injected into the tactile device using the psychophysical model presented in Equation 1.

\section{One dimensional case}

The one-dimensional case is used for defining a tactile profile along a single axis as employed in the pairwise experiment discussed above, and was used in the research presented in $[17,21]$. The gradient of the surface $F(x)$ is:

$$
\nabla F(x)=d F(x) / d x
$$

Let's assume that the finger slides on the touch screen in the positive $x$-direction. The gradient is normalized by taking the dot product of the gradient and the unit vector along the direction of finger motion. This normalized gradient is scaled to the friction force level and then mapped to the applied voltage using the perceptual model defined by Equation 1 and injected into the tactile feedback device.

\section{Generalized two-dimensional case}

A growing body of haptics research utilizes image-based haptic rendering techniques $[2,26]$. The purpose of generalization is to accommodate a wide variety of inputs to be rendered using our algorithm.

As an example, we implement the algorithm on a twodimensional depth map, that can be computed from a 3D model or obtained from a depth camera. The input is a typical 2D grayscale depth image (Figure 8(a)) and a user slides the finger across it. The procedure can be easily extended to other use cases, such as mathematical models for texture synthesis [26].

Step 1: Calculate the $2 D$ gradient field

From the 2D grayscale image, we calculate the gradient of the depth distribution, which is defined as:

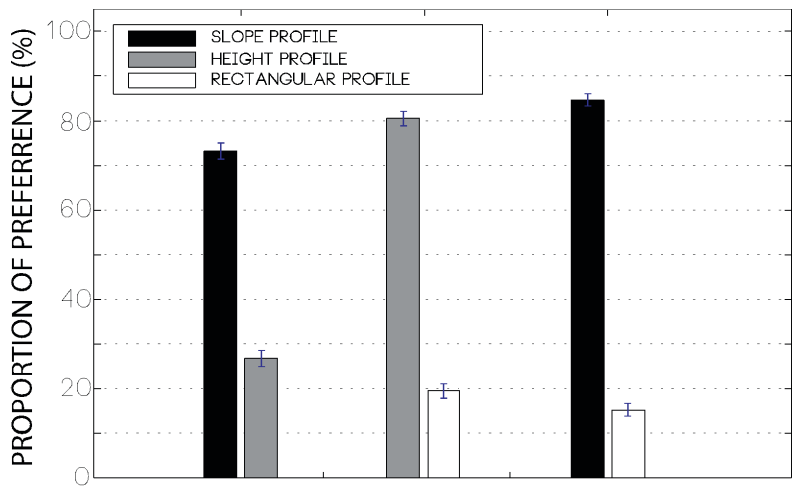

Figure 6: Results of pairwise comparison experiment

$$
\nabla F(x, y)=\left(\frac{\partial F}{\partial x}, \frac{\partial F}{\partial y}\right)=\frac{\partial F}{\partial x} \hat{i}+\frac{\partial F}{\partial y} \hat{j}
$$

where $F(x, y)$ is the two-dimensional depth distribution. This computation is typically done as a preprocessing step and yields a $2 \mathrm{D}$ vector field in $x y$ plane. Each vector points in the direction of increasing depth of $F$, i.e. represents it steepest ascent Figure 8(b) shows the gradient fields along the $x$ - and $y$-axis. Figure 8(c) shows the 2D image overlaid with levels of haptic feedback as the user slides the finger on a marked path.

Step 2: Determine the user's finger motion on touch screen Define a unit direction vector of the finger motion in contact with the touch screen as:

$$
\overline{\mathbf{v}}_{i}=\frac{\mathbf{x}_{i}-\mathbf{x}_{i-1}}{\left|\mathbf{x}_{i}-\mathbf{x}_{i-1}\right|}
$$

where $\mathbf{x}_{i}$ is the finger position vector at the time of $t_{i}$. We usually compute a moving average of $\overline{\mathbf{v}}$ in case the touch screen has a low spatial resolution.

\section{Step 3: Calculation of perceptual friction force}

Compute the interaction between the static gradient field $\nabla F$ computed at Step 1 and user finger motion $\overline{\mathbf{v}}$ as

$$
f_{i}=f_{0}|\nabla F| \cos \theta_{i}=f_{0} \nabla F \cdot \overline{\mathbf{v}}_{i} .
$$

Where, $f_{i}$ is the scalar value representing the perceptual frictional force at a given instant $t_{i}, f_{0}$ is a non-zero scale factor, and $\theta_{i}$ is the angle between two vectors.

Step 4: Calculation of controlled voltage for the device Use the model in Equation 1 to compute the control voltage

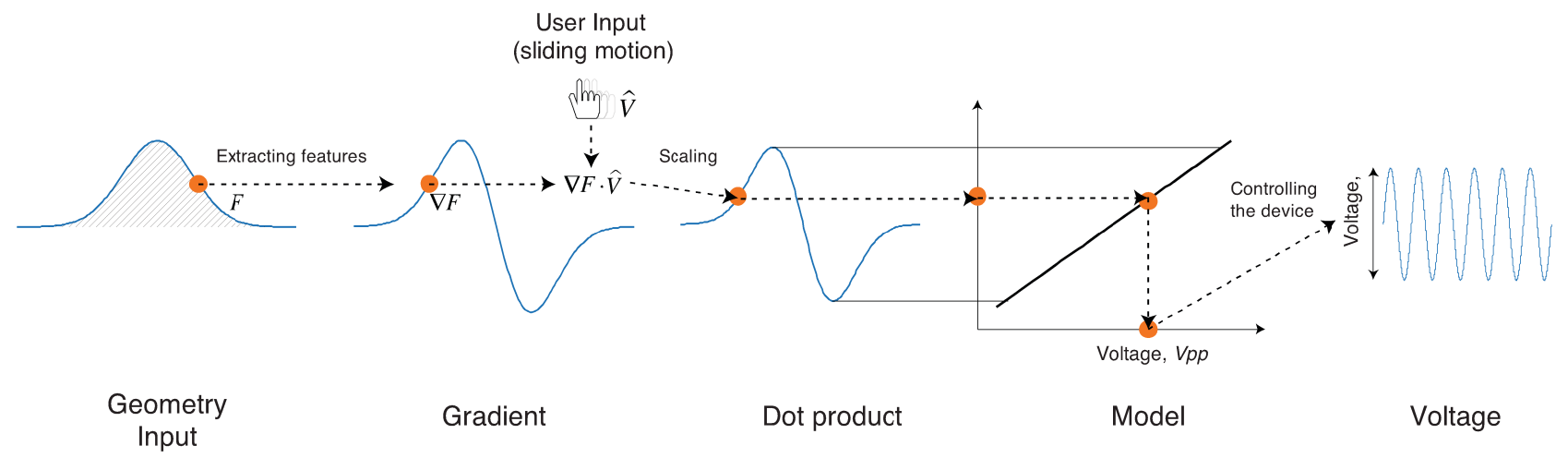

Figure 7: A block diagram summarizing of the tactile rendering algorithm. 


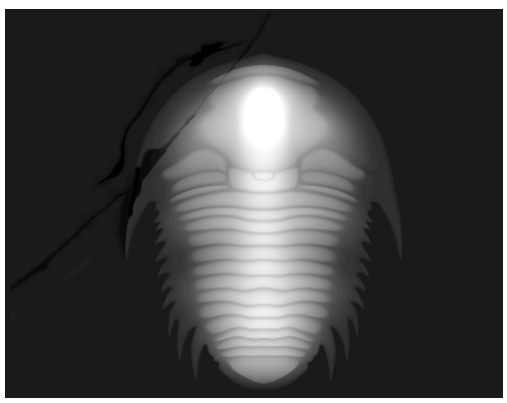

(a)

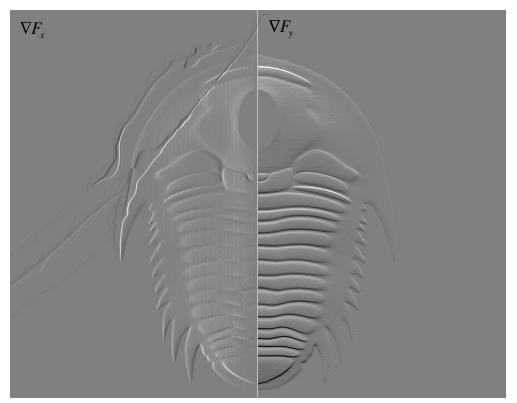

(b)

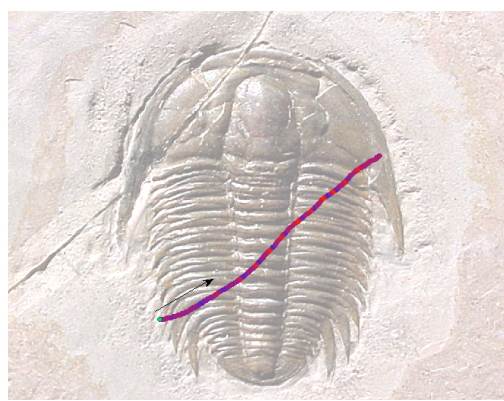

(c)

Figure 8: (a) Depth map (input image) extracted from a 3D model, (b) Gradient in the $x$ - and $y$ - direction, $\nabla F_{x}, \nabla F_{y}$, and (c) The actual 2D image overlaid with the haptic feedback rendered with our algorithm as the user moved in a shown path.

that corresponds to the required friction force $f$ computed in Equation 5. In order to utilize the entire range of the device, we can scale friction force values to the range between 0 and 100 corresponding to the minimum and the maximum gradient values of the given input image.

The proposed algorithm is easily extended to render tactile feedback for other forms of input. It can also be easily incorporated into other types of friction-based lateral tactile displays.

\section{Explorations and applications}

In this section, we explore the use of our tactile rendering algorithm to create realistic 3D tactile features on a touch screen for a broad variety of images incorporating depictions of everyday objects, textures, patterns, etc. The following scenarios explore the further uses of the algorithm, and are not in any way limiting its use in other applications.

\section{Depth maps}

The two-dimensional depth image is one of the most common media used for defining haptic fields [26]. A typical example is its use with the 3D model as shown in Figure 9(a). The 3D information encoded in depth fields is widely used in various types of interactive applications, such as games, navigation, maps, computer graphics, etc. With our algorithm we can enhance depth maps with 3D tactile sensations.

Depth images can be also acquired directly from depthmeasuring cameras, which are widely employed in various HCI contexts with the introduction of Microsoft Kinect. Figure 9(b) shows an example of interaction with a depth map stream taken from Kinect. This can be further applied to the systems for the visually impaired, where the direct video stream can be used to render environmental conditions.

\section{Tactile content authoring}

Previous studies have addressed the importance of haptic
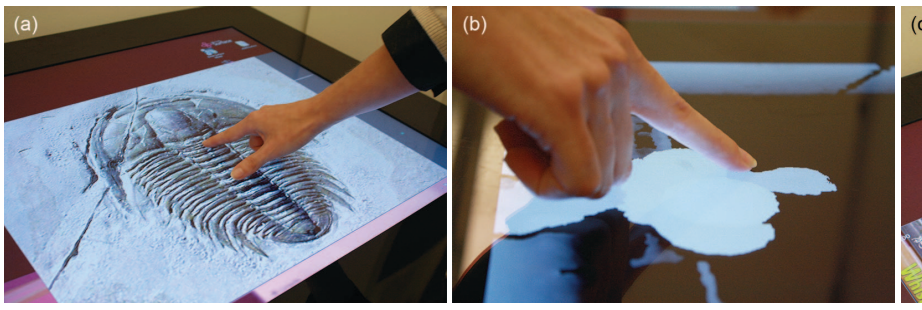

feedback in touch interaction [2]. With this in mind, we applied the proposed algorithm to enhance visual information, by overlaying haptic content on digital pictures. Figure 9(c) shows an example where the user selects books from the virtual library and can feel spine covers rendered as 3D tactile bumps. The tactile experiences can be dynamically adjusted according to information associated with the object in the picture. For example, book thickness or user book preferences can be mapped to different parameters of the Gaussian bump profile that is used for books tactile rendering.

Tactile UI

Oakley et al. [18] augmented the standard GUI components with the haptic effects, such as haptic recess effect, snap-to effect (gravity well), friction, and tactile textures. Similarly, we added 3D tactile bumps to standard GUI components. Unlike in previous work, however, we render 3D tactile bumps algorithmically and can dynamically change their properties depending on changes in the GUI elements. We expect this approach can be used not only for enriching touch experiences, but also to increase accessibility of the standard GUI components.

\section{$3 D$ graphical models}

The algorithm can be also applied to $3 \mathrm{D}$ models commonly produced in the computer graphics communities. Because the gradient, $\nabla F$, is equivalent to the normal vector of the constructed mesh surface, the Equation 5 can be written as:

$$
f=f_{0} \overline{\mathbf{n}} \cdot \tilde{\mathbf{v}} \text {. }
$$

Where, $\overline{\mathbf{n}}$ is a $1 \times 3$ unit normal vector of the mesh that is being touched by the user and $\tilde{\mathbf{v}}$ is the augmented $1 \times 3$ movement direction vector, $[\overline{\mathbf{v}} \mid 0]^{\mathrm{T}}$. Thus, arbitrary 3D models and the entire dynamic 3D scenes and animations can be $3 \mathrm{D}$ haptically rendered in real time, without the need to pre-compute gradient field in advance.
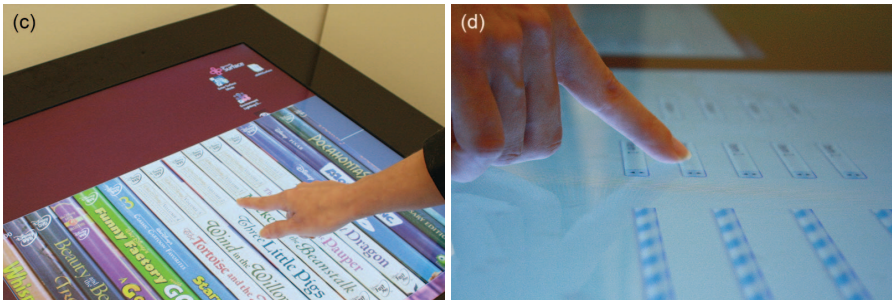

Figure 9. Applications of 3D tactile bumps in various interaction scenarios. 


\section{Haptic augmented reality}

AR markers and other tracking techniques can be used to obtain the gradients of the geometric field corresponding to the real world around the user. Consequently, the depth and gradient field of the objects and surfaces can be computed and their tactile representations can be rendered using or algorithm.

\section{CONCLUSIONS}

In this paper, we present a tactile-rendering algorithm that simulates geometric features such as bumps on flat touch surfaces using only lateral resistance forces produced by the electrovibration effect. We have evaluated algorithm in controlled experimental studies that demonstrated that we can create a realistic and convincing tactile sensations of $3 \mathrm{D}$ bumps on a variety of visual content, including photos and $3 \mathrm{D}$ models. This functionality can be easily incorporated into touch-based interfaces and allow for the creation of effective and engaging tactile user experiences.

\section{REFERENCES}

1. Bau, O. and Poupyrev, I. REVEL: tactile feedback technology for augmented reality. ACM Transactions on Graphics (TOG), 31, 4 (2012), 89.

2. Bau, O., Poupyrev, I., Israr, A. and Harrison, C. TeslaTouch: electrovibration for touch surfaces. In Proc. UIST '10, ACM (2010), 283-292.

3. Brewster, S., Chohan, F. and Brown, L. Tactile feedback for mobile interactions. In Proc. CHI'07, ACM (2007), 159-162.

4. Casiez, G., Roussel, N., Vanbelleghem, R. and Giraud, F. Surfpad: riding towards targets on a squeeze film effect. In Proc. CHI'11, ACM (2011), 2491-2500.

5. Geldard, F. A. Adventures in tactile literacy. American Psychologist, 12, 3 (1957), 115.

6. Gibson, J. J. Observations on active touch. Psychological review, 69, 6 (1962), 477-491.

7. Gordon, I. E. and Morison, V. The haptic perception of curvature. Attention, Perception, \& Psychophysics, 31, 5 (1982), 446-450.

8. Hoggan, E. and Brewster, S. New parameters for tacton design. In Proc. CHI '07 Extended Abstract, ACM (2007), 2417-2422.

9. Israr, A. and Poupyrev, I. Tactile brush: drawing on skin with a tactile grid display. In Proc. CHI'11, ACM (2011), 2019-2028.

10. Lévesque, V., Oram, L. and MacLean, K. Exploring the Design Space of Programmable Friction for Scrolling Interactions. In Proc. IEEE Haptics Symposium (2012), 24-30.

11. Lévesque, V., Oram, L., MacLean, K., Cockburn, A., Marchuk, N.D., Johnson, D., Colgate, J.E. and Peshkin, M.A. Enhancing physicality in touch interaction with programmable friction. In Proc. CHI'11, ACM (2011), 2481-2490.

12. Lederman, S. and Klatzky, R. Haptic perception: A tutorial. Attention, Perception, \& Psychophysics, 71, 7 (2009), 14391459.

13. Lederman, S. J. and Klatzky, R. L. Hand movements: a window into haptic object recognition. Cognitive psychology, 19, 3 (1987), 342-368.
14. Lederman, S. J. and Klatzky, R. L. Haptic perception: A tutorial. Attention, Perception, \& Psychophysics, 71, 7 (2009), 1439-1459.

15. Lee, J. C., Dietz, P. H., Leigh, D., Yerazunis, W. S. and Hudson, S. E. Haptic pen: a tactile feedback stylus for touch screens. In Proc. UIST '04, ACM (2004), 291-294.

16. MacLean, K. E. Haptic interaction design for everyday interfaces. Reviews of Human Factors and Ergonomics, 4, 1 (2008), 149-194.

17. Minsky, M., Ming, O.-y., Steele, O., Frederick P. Brooks, J. and Behensky, M. Feeling and seeing: issues in force display. SIGGRAPH Comput. Graph., 24, 2 (1990), 235-241.

18. Oakley, I., McGee, M. R., Brewster, S. and Gray, P. Putting the feel in 'look and feel'. In Proc. CHI '00, ACM (2000), 415-422.

19. Poupyrev, I. and Maruyama, S. Tactile interfaces for small touch screens. In Proc. UIST '03 (2003), 217-220.

20. Poupyrev, I., Rekimoto, J. and Maruyama, S. TouchEngine: a tactile display for handheld devices. In Proc. CHI '02 Extended Abstract, ACM (2002), 644-645.

21. Robles-De-La-Torre, G. and Hayward, V. Force can overcome object geometry in the perception of shape through active touch. Nature, 412, 6845 (2001), 445-448.

22. Romano, J. M. and Kuchenbecker, K. J. Creating Realistic Virtual Textures from Contact Acceleration Data. Haptics, IEEE Transactions on, 5, 2 (2012), 109-119.

23. Rosenberg, L. B. Virtual fixtures: Perceptual tools for telerobotic manipulation. In Proc. Virtual Reality Annual International Symposium, 1993., 1993 IEEE (1993), 76-82.

24. Saga, S. and Deguchi, K. Lateral-Force-Based 2.5Dimensional Tactile Display for Touch Screen. In Proc. IEEE Haptics Symposium (2012).

25. Salisbury, K., Brock, D., Massie, T., Swarup, N. and Zilles, $\mathrm{C}$. Haptic rendering: programming touch interaction with virtual objects. In Proc. Proceedings of the 1995 Symposium on Interactive 3D graphics, ACM (1995), 123-130.

26. Srinivasan, M. and Basdogan, C. Haptics in virtual environments: Taxonomy, research status, and challenges. Computers \& Graphics, 21, 4 (1997), 393-404.

27. Weiss, M., Wacharamanotham, C., Voelker, S. and Borchers, J. FingerFlux: near-surface haptic feedback on tabletops. In Proc. UIST '11, ACM (2011), 615-620.

28. Wijekoon, D., Cecchinato, M., Hoggan, E. and Linjama, J. Electrostatic Modulated Friction as Tactile Feedback: Intensity Perception. In Proc. Eurohaptics Springer Berlin Heidelberg (2012), 613-624.

29. Winfield, L., Glassmire, J., Colgate, E. and Peshkin, M. TPaD: Tactile Pattern Display through Variable Friction Reduction. In Proc. World Haptics (2007), 421-426.

30. Yohanan, S. and MacLean, K. E. Design and assessment of the haptic creature's affect display. In Proc. HRI '11, ACM (2011), 473-480.

31. Zwislocki, J. J. and Goodman, D. A. Absolute scaling of sensory magnitudes: A validation. Attention, Perception, \& Psychophysics, 28, 1 (1980), 28-38. 